\title{
La diversidad sexo-genérica: Un punto de vista evolutivo
}

\author{
Rafael J. Salín-Pascual'
}

Actualización por temas

\section{ABSTRACT}

\section{Introduction}

There are people with a gender incoherence between their biological gender (phenotype) and the self-perception gender. Differences among such condition and sexual orientation give us more subtypes of gender and sexual diversity: transsexual, travesties and transgender, homosexual, heterosexual, bisexual and asexual.

\section{Objective}

To review if there are well supported evidences about sexual and gender diversity as part of evolutionary strategies.

\section{Method}

Medical and political historical documents about the birth of the concepts of gender and sexual orientation were consulted at The National Library of Medicine. These were updated, in a review of the scientific literature of the last fifty years in SCOPUS, PubMed and Science Direct systems. The following words were used: homosexuality, transsexuality, gender and evolution.

\section{Results}

Because sexual reproduction is so indispensable and so zealously selected, the existence of homosexuality and transsexuality is a kind of paradox. One must wonder: why would not evolution quickly select against behavior, which diverts an animal from sexual reproduction? Yet despite this apparently unlikelihood homosexuality does exist.

Homosexuality is also the innate sexual preference for one's own gender or the biological urge for same-sex coitus. So despite popular non-recognition of the phenomenon, natural history observations have revealed a wide range of homosexuality throughout the animal kingdom.

To account for homosexuality -or any phenomenon- using evolution, it is necessary that it be natural, i.e. it must occur naturally without human influence. Thus, animal behavior is used to illustrate the naturalness of homosexuality. In essence: there are homosexual animals in nature; therefore homosexuality is natural.

Transsexuality is a gender issue, and in psychiatry remains as a mental disease named "gender dysphoria".

\section{Discussion and conclusion}

There is some biological basis for these particular human beings, in whom the role of high levels of hormones, antibodies against testosterone receptors, order or birth is also discussed in the present article.

To understand that humans are not a dichotomist species is the main goal of this work, as homo sapiens differences in many aspects of our functions are the norm.

Key words: Transsexual, homosexual, gender, evolution, homophobia.

\section{RESUMEN}

\section{Introducción}

Hay un grupo de gente con una incoherencia entre el género y su sexo biológico (fenotipo) con el género de autopercepción. Las diferencias entre esta condición y la orientación sexual nos dan una serie de subtipos de género y de diversidad sexual: transexuales, travestis y transgéneros, homosexuales, heterosexuales, bisexuales y asexuales.

\section{Objetivo}

Revisar las evidencias que puedan apoyar si la diversidad de género y la orientación sexual son estrategias evolutivas.

\section{Método}

Se consultaron documentos históricos, médicos y políticos sobre e nacimiento de los conceptos de género y orientación sexual en The National Library of Medicine. Estos se actualizaron en una revisión de la literatura científica de los últimos cincuenta años en los sistemas SCOPUS, PubMed y Science Direct. Se utilizaron las palabras: homosexuality, transsexuality, gender y evolution.

\section{Resultados}

Debido a que la reproducción sexual es tan indispensable y celosamente seleccionada, para continuar la combinación genética, la existencia de la homosexualidad y la transexualidad son una paradoja evolutiva. Hay una gama de este tipo de conductas en los animales de reproducción sexual, en mamíferos, aves, reptiles y peces.

Hasta el momento sólo hemos utilizado el comportamiento animal para ilustrar la naturalidad de la homosexualidad. En esencia: hay animales homosexuales en la naturaleza. Por lo tanto, la homosexualidad es natural, y en este artículo se presentan las explicaciones evolutivas al respecto.

La transexualidad es una cuestión de género, y en la psiquiatría contemporánea sigue siendo calificada como una enfermedad mental, llamada "disforia de género".

\section{Discusión y conclusión}

Hay bases biológicas para esta alternativa particular en los seres humanos, en los que el papel de los niveles de hormonas elevados, los anticuerpos contra los receptores de testosterona, el orden del nacimiento y el uso de algunas drogas se discuten en el presente artículo.

Comprender que los seres humanos no son una especie dicotómica es el objetivo principal de este trabajo, ya que en el homo sapiens, las diferencias en muchos aspectos de nuestras funciones es la norma que nos hace tan diferentes, pero al mismo tiempo iguales en derechos básicos como seres humanos.

Palabras clave: Homosexualidad, transexuales, género, evolución, homofobia.

Departamento de Psiquiatría y Salud Mental. Facultad de Medicina. Universidad Nacional Autónoma de México.

Red Interinstitucional de Atención a la Diversidad Sexual. Gobierno del Distrito Federal, México.

Clínica de la Diversidad Sexual (DIVERGEN-UNAM). Facultad de Medicina. Universidad Nacional Autónoma de México.

Correspondencia: Doctor Rafael J. Salín-Pascual. Hidalgo 187 casa 7, Barrio del Niño Jesús, Tlalpan, 04100 , México DF. E-mail: rafasalin@yahoo.com, rafasalinpas@gmail.com

Recibido: 16 de junio de 2014. Aceptado: 25 de septiembre de 2014 
"Visto a la luz de la evolución, la biología es, quizás, la ciencia más satisfactoria e inspiradora. Sin esa luz, se convierte en un montón de hechos varios, algunos de ellos interesantes o curiosos, pero sin formar ninguna visión conjunta."

Theodosius Dobzhansky

\section{INTRODUCCIÓN}

Hay personas con una incoherencia entre su sexo biológico (fenotipo) y la percepción de su género, que puede ser exactamente el opuesto al que se les otorgó al nacer. Las diferencias entre estas dos condiciones, sexo y género, nos proporcionan más subdivisiones, tales como transexuales, travestis y transgéneros, homosexuales, bisexuales, heterosexuales y asexuales. ${ }^{1}$

Las personas transexuales (PTS) presentan una condición en etapas tempranas de su vida, cuando reconocen tener alguna incoherencia acerca de cómo se perciben ellos mismos. Esto es, que son incoherentes con su apariencia externa, en la manera en cómo la gente reacciona ante ellos, y cómo se perciben ellos mismos, lo cual es en sentido opuesto a como lo hacen los otros. Por lo general, las PTS piden ser nombrados y abordados por sus apellidos o por un nombre que corresponde al opuesto al de su género asignado al nacer. ${ }^{2}$

Esta incoherencia sexo-genérica, vista de manera natural, nos brinda un estado de discordancia entre el modo en que aparece externamente una persona (fenotipo, con ambas facetas, biológica y social), y la percepción del "uno-mismo" o autopercepción. Esta última, es una función neurobiológica que vamos generando desde la etapa intrauterina. La información propioceptiva, que proviene de extremidades, tronco y cabeza, va creando una zona de integración en una de las grandes áreas de asociación, ubicada en la unión de los lóbulos parietal, temporal y occipitales, del lado derecho. . $^{3,4}$ Esta autopercepción se estructura biológicamente y tiene un constructo continuo con la crianza, los papeles estereotipados asignados a los géneros, y se va consolidando y complementando hasta la pubertad. En cierto sentido, esto es lo que nos proporciona una continuidad ontológica. Factores como los estereotipos de la crianza, la asignación de nombres, papeles en juegos infantiles, colores en las vestimentas, tipo de educación escolar e interacción con los adultos y compañeros de infancia, determinan que nos identifiquemos dentro de un género en particular. ${ }^{5}$ Los mecanismos del tipo imitación y empatía, en los que participan las neuronas en espejo, que remedan y graban la información de conductas, ademanes, estilos de marcha y locución, nos muestran cómo el niño varón imita al padre y la niña, a la madre. ${ }^{6}$

Los primeros indicios de una incoherencia sexo-genérica, se ven cuando ocurre lo contrario; es decir, que el niño adopta modales de la madre y la niña los del padre. Esto, por lo general, es aceptado pocas veces por los padres, incluso es negado o, en el peor de los casos, reprimido. ${ }^{2}$
De la genética y de sus expresiones como el fenotipo se desprende que puedan ser dos pares de cromosomas bien definidos los responsables del fenotipo sexo-genérico ( $X X$ o $X Y$, para hembras y varón, respectivamente). El sexo gonadal y fenotípico, como hombre o mujer, se correlaciona con esta primera división dicotómica. Las personas intersexuales son la excepción a lo dicho previamente, ya que pueden tener un exceso: $X X X, X X Y$, o un déficit, $X O$, de los cromosomas sexuales. ${ }^{7}$ Sobre el sexo psicológico, los hombres y las mujeres son diferentes en virtud de su propia identidad de género, lo que significa que se reconocen como pertenecientes a un género específico, por lo general coherente con su aspecto externo. ${ }^{8,9}$

Cualquiera que sea la orientación sexual, su condición de género o fenotipo biológico, ésas son las variaciones humanas de una función fisiológica específica y particular: la actividad sexual sin la necesidad de reproducción. Esta posición es subversiva de entrada y se contrapone a los valores morales y religiosos que se asentaron en la burguesía a partir del siglo XVIII. ${ }^{10}$ La parte hedónica de la actividad sexual es propia de los primates y tiene especial relevancia en tres de ellos: bonobos, chimpancés y Homo sapiens. La variación del genoma en estas tres especies está entre el 2\% y el 3\%. ${ }^{11}$

La función reproductiva no es la meta principal en la actividad sexual humana, sino la de mantener el deseo sexual activo y funcional. Esto se ha logrado aun cuando exista una ausencia de hembras, o bien, cuando las que existen en dichas comunidades no fueran capaces de reproducirse. ${ }^{11}$

La reproducción no está cancelada en personas homosexuales o transexuales, aunque no es una meta en sus vidas. Esta observación ha sido relevante para los propósitos evolutivos. ¿Por qué la diversidad sexual y de género se expresan en todo el mundo en tasas constantes, aun cuando la reproducción entre dichas personas no sea algo común? ¿Por qué las personas de la diversidad LGBTTTI (lesbianas, gays, bisexuales, transgéneros, transexuales, travestis e intersexuales) no disminuyen en prevalencia? Las estrategias evolutivas que persisten en una especie en particular deben ser vistas como parte del marco de referencia evolutivo y como parte del mecanismo general de adaptación. Hay una gran cantidad de ejemplos de tales diferencias de sexo y de género en el reino animal. ${ }^{12-15}$

Una mosca de la fruta, por ejemplo, puede cortejar a otros machos porque carece de un gen que le permite establecer la diferencia entre los sexos. Pero eso es muy diferente a los delfines de nariz de botella machos, que se dedican a las interacciones entre individuos del mismo género para facilitar la unión de grupo, o el albatros de Laysan femeninos, que pueden permanecer consolidados de por vida $y$ desarrollan funciones cooperativas en la crianza de los polluelos. ${ }^{16}$ Pocos estudios han examinado si las relaciones del mismo sexo participan activamente en los procesos evolutivos de una manera específica. Ésta es la propuesta de conductas que persisten a lo largo de la evolución. El que tienen una utilidad y por lo tanto se preservan. 
Las conductas sexuales con el mismo género -el cortejo, la conducta de monta o la crianza de los hijos- son rasgos que pueden haberse formado por selección natural, por un mecanismo básico de evolución, que se produce a través de generaciones sucesivas. Sin embargo, nuestra revisión de los estudios sugiere que estas conductas sexuales con el mismo género pueden actuar como fuerzas selectivas en sí mismas. En otras palabras, las relaciones homosexuales pueden contribuir a la evolución de maneras sutiles y alternas y no menos importantes para muchos animales. Cuando los biólogos piensan en la presión selectiva en la evolución, tienden a centrarse en las preocupaciones ambientales, tales como el clima, la temperatura o accidentes geográficos en una localidad particular. Sin embargo, las circunstancias sociales también pueden tener un impacto, ${ }^{14}$ como las relaciones entre personas del mismo género, que podrían cambiar radicalmente las circunstancias sociales; por ejemplo, mediante la eliminación de algunos individuos del grupo de animales disponibles para el apareamiento. La competencia y selección reproductiva en los primates se hace en la vagina de la hembra, que al ser receptora posible de múltiples machos, desarrolló mecanismos de selección natural en función de la cantidad de espermatozoides y su potencia, de la etapa del ciclo sexual de la hembra, y de otros factores que llevan a la optimización de la reproducción y sobrevivencia de los infantes. ${ }^{17,18}$

También se ha estudiado al albatros Laysan, una especie en la que las hembras forman parejas del mismo género y crían juntas a los polluelos de una de ellas o de ambas. El comportamiento del mismo género, en esta especie, puede no ser aberrante, pero en cambio puede surgir como una estrategia reproductiva alternativa. Casi un tercio de las parejas de albatros de Laysan son pares hembra-hembra y tienen más éxito que las hembras no apareadas cuando se trata de la crianza de los polluelos..$^{19,20}$ Los comportamientos sexuales entre individuos del mismo género son desplegados flexiblemente en una variedad de circunstancias. Por ejemplo, en las tácticas reproductivas alternativas, en las estrategias de mejoramiento de cooperación, como facilitadores de la vinculación social y como mediadores de conflictos intersexuales. Una vez establecida esta flexibilidad, se convierte, en sí misma, en una fuerza selectiva que puede llevar a la selección en otros aspectos de la fisiología, historia de vida, y del comportamiento social.

\section{LA PARADOJA HOMOSEXUAL Y TRANSEXUAL DESDE EL PUNTO DE VISTA EVOLUTIVO}

Mientras que algunas especies de plantas y animales se reproducen por ambos tipos de reproducción, asexual y sexual, muchas especies son estrictamente asexuales. Por medio de este tipo de reproducción, se logran clonaciones de sí mismas. Todos los individuos de una población comparten una estructura genética idéntica. Esta forma de reproducción tiene varias ventajas. Es simple desde los puntos de vista conductuales, fisiológicos y morfológicos. Por lo tanto, hay menos posibilidad de error. La reproducción asexual ocurre en los organismos que están bien adaptados a un entorno en particular y que no tienen que diluir sus genes, ya acondicionados a partir de otros padres potencialmente menos acoplados. Las especies con reproducción asexual no tienen que gastar energía para buscar, atraer y cortejar a la pareja. Tampoco necesitan evolucionar los comportamientos especializados ni las características físicas para llevar a cabo estos cortejos, como tener cornamentas fuertes y masivas, presumir cantos complejos, gruñidos intimidantes, ni construir nidos ad hoc para que la hembra empolle los huevos. Tampoco desarrollar un estado de bienestar económico que atraiga a las hembras de nuestra especie. ${ }^{6}$

Por razones como éstas, la existencia misma de las relaciones sexuales es un "complejo rompecabezas". A decir verdad, la mayoría de las criaturas estarían mejor sin esta actividad, que consume gran parte de su energía y vida. Pero ¿por qué es necesario el sexo? Porque el sexo hace posible la recombinación y perpetuación de códigos genéticos que han impulsado la evolución, y suprime las mutaciones deletéreas para los nuevos individuos. Mientras que la evolución es la fuerza fundamental de la biología, el sexo es la argamasa que mantiene esta fundación unida. Así de ventajoso es el sexo. A pesar del alto coste biológico, la mayoría de los animales macroscópicos -incluyendo el 99\% de todos los vertebrados- se reproducen sexualmente. ${ }^{12}$

Lo cierto es que la reproducción sexual es tan indispensable y tan celosamente seleccionada para la existencia, que la homosexualidad es una paradoja. La homosexualidad parece ser la antítesis de la reproducción sexual, el enemigo de la evolución. Uno debiera de preguntarse: ¿por qué la evolución seleccionó una estrategia en contra de la reproducción? Sin embargo, a pesar de que parece que la homosexualidad es contraria a la evolución, no lo es, al menos no en un sentido real, pues existe, y no es un capricho, sino que cumple varias funciones evolutivas. ${ }^{21}$ Para empezar, es una conducta que está muy extendida en todas las especies con reproducción sexual en animales. ¿Por qué?

\section{LA DEFINICIÓN DE LA HOMOSEXUALIDAD}

Para responder a la pregunta anterior, primero tenemos que identificarla. "La homosexualidad" tiene dos significados principales, que son distintos pero relacionados. La homosexualidad es la actividad sexual entre dos personas del mismo género. Esto incluye el coito entre personas del mismo género o contacto genital, ya sea ventral-ventral, dorso-ventral, o de otras maneras, ya sea hombre-varón o mujer-hembra. 
La homosexualidad es también la preferencia sexual innata por el propio género, o el impulso biológico para tener coitos entre personas del mismo género. Si bien se habla mucho de la distinción entre los deseos homosexuales (o deseo sexual innato) y el comportamiento homosexual en los seres humanos, esta distinción es de importancia limitada o insignificante en poblaciones no humanas, en las que inferimos "el deseo" (ya sea instintivo o aprendido) primario con base en el comportamiento. Hay un abismo entre el deseo humano/impulso biológico y el comportamiento de los animales. ${ }^{22}$ El deseo y la conducta sexual en humanos se atribuyen a las costumbres humanas sociales y culturales. ${ }^{16}$ En resumen, la homosexualidad es, a la vez, la unidad biológica para el deseo del coito entre personas del mismo género (intensión de la actividad), así como la realización del coito entre estas personas (llevar a cabo dicha actividad). La homosexualidad se ha observado en los machos y hembras, tanto en las poblaciones humanas como en las no humanas. Si bien es difícil, si no imposible, separar impulso innato de la acción en los animales no humanos, la distinción entre estas dos definiciones será importante más adelante mientras discutimos posibles explicaciones evolutivas para la homosexualidad. ${ }^{23,24}$

\section{LA HOMOSEXUALIDAD NEGADA}

En muchas especies de primates, la monta entre individuos del mismo género es más que incidental y no debe afirmarse que es una aberración. ${ }^{25}$ En los bonobos femeninos se ha observado que eligen el roce genital-genital con otras hembras durante la cópula con un macho. Durante una temporada en la que se observaron a 58 hembras bonobo, 45 de ellas estaban más comprometidas en la actividad homosexual y algunas eran exclusivamente homosexuales. Se observó un total de 98 montas homosexuales en los gorilas de montaña masculinos; éstos eran encuentros ventro-ventral y dorsoventral. ${ }^{26}$

Otros mamíferos presentan conductas homosexuales. Por ejemplo, hay una proclividad para la monta en el ganado bovino hembra-hembra; esto ha sido notado por los productores de leche, quienes velan por este tipo de conducta como indicador de la fase de estro. La conducta de monta macho-macho es un comportamiento típico de las ovejas de montaña. ${ }^{26}$ La conducta homosexual también se ha observado en las jirafas, ratas, delfines, perros, hembras de ciervos, burros, gatos, carneros, cabras, cerdos, antílopes, elefantes, hienas, conejos, lobos, puerco espines, hámsters, ratones y marsopas. ${ }^{26}$

Los juegos homosexuales son una de las conductas más detectadas en mamíferos. Se llevan a cabo la monta entre sí de manera indiscriminada, y esto lleva a la consolidación de los grupos. ${ }^{25}$ Ciertamente, la actividad homosexual en el adulto es más pertinente, como un entrenamiento o práctica para el éxito reproductivo real, pero el comportamiento de los menores podría ser indicativo de la naturaleza innata del deseo sexual en general. Varios otros ejemplos de este tipo de actividad se han registrado en aves, reptiles, peces e insectos. Es impresionante cómo la moralidad de una cultura crea una serie de filtros a conductas tan diseminadas en los seres vivos.

\section{EVOLUCIÓN HOMOSEXUAL}

A partir de la existencia de la homosexualidad en la naturaleza y de su frecuente agrupación taxonómica (es decir, los organismos más estrechamente relacionados que muestran un comportamiento homosexual cada vez más similar) se puede inferir que la homosexualidad ha evolucionado. Pero debido a que nadie ha sido capaz de observar la evolución de la homosexualidad de primera mano, sólo podemos teorizar sobre cómo sucedió. Con base en estudios de comportamiento de la homosexualidad en el ser humano y los animales, se han propuesto una serie de modelos y explicaciones sobre cómo se ha desarrollado esta práctica aparentemente acoplada a la expresión genética. Hay dos tipos de explicaciones principales de cómo y por qué la homosexualidad se observa en los animales: ${ }^{26}$

1. Los comportamientos homosexuales (como la conducta de monta a individuos del mismo género y la mímica de género cruzado) no son funcionales, pero los efectos secundarios no son particularmente dañinos a la conducta adaptativa. La homosexualidad, entonces, no es intencional (es decir, el animal no tiene utilidad para la cópula entre individuos del mismo género).

2. La homosexualidad es adaptativa, ampliando la capacidad de un organismo para transmitir sus genes. La homosexualidad es, por lo tanto, intencional (es decir, el animal tiene una unidad específica para la cópula entre personas del mismo género).

Los biólogos, quienes por lo general estudian un rango limitado de animales, han gravitado hacia una u otra de estas dos explicaciones $\mathrm{y}$, a veces, han extrapolado sus teorías para explicar en términos generales la homosexualidad animal. Sin embargo, al evaluar el amplio espectro de diferentes comportamientos homosexuales en el reino animal, se ve claramente que la homosexualidad se ha desarrollado tanto como un efecto secundario, como una conducta de adaptación ventajosa.

\section{LA HOMOSEXUALIDAD COMO UN EFECTO SECUNDARIO}

Se ha descrito el modelo del efecto secundario de la evolución de la homosexualidad. ${ }^{14}$ En él se propone que la homosexualidad sea el resultado de la intensa necesidad por tener 
actividad sexual. El funcionamiento de este modelo tiene mucho que ver con los principios de la heterosexualidad. En los mamíferos, las hembras deben llevar a sus crías durante el embarazo, lo que limita su potencial número de descendientes. Los hombres sólo necesitan contribuir con esperma, sin embargo, no pueden tener ninguna inversión adicional en el tiempo en que se gestan sus hijos. Los machos pueden producir espermatozoides, con eficacia casi sin fin y ser padres de muchos descendientes. El éxito reproductivo de un mamífero macho se ve reforzado cuando insemina a muchas hembras, lo que garantiza un mayor número de crías. Pero una mujer no puede producir un mayor número de crías mediante el aumento de la frecuencia de cópulas, por lo que no es beneficioso para una mujer el aumentar su número de parejas sexuales. Una hembra aumenta su aptitud reproductiva mediante la selección del mejor padre posible para su descendencia; esto amplía la aptitud y la posibilidad de supervivencia en sus crías. Gran parte de la diferencia entre el comportamiento sexual masculino y el femenino en los mamíferos, se puede explicar simplemente por el hecho de que las hembras están fuera de la lotería genética durante el tiempo del embarazo.

Gracias a la evolución, los hombres han adquirido un comportamiento que les obliga a competir por las hembras con otros machos. Esto último les lleva a tener cópulas más frecuentes y, por lo tanto, logrará un mayor éxito reproductivo. El modelo de efectos secundarios de la homosexualidad propone que "la hipersexualidad" masculina les lleva a montar a una serie de hembras y machos. Este exceso de deseo sexual masculino se ha propuesto como una estrategia para fecundar más hembras y mantener el deseo sexual activo, funcional y facilitado. * Si por razones de muerte en el trabajo de parto $\mathrm{u}$ otras causas no hay suficientes mujeres en una comunidad, una actividad practicada por primates en general (chimpancés y Homo sapiens), es el rapto de hembras en ataques sorpresivos a otros clanes. En caso de que esto no sea probable, se agudizará la actividad homosexual. ${ }^{21}$

Esta teoría se encuentra bien apoyada por las observaciones de la conducta sexual en el reino animal. Como se ha señalado, en los animales la "homosexualidad" es un término equivocado, pues realmente se refiere a la bisexualidad o "ambisexualidad", o al comportamiento de monta con los animales de ambos géneros. Sólo hay unas pocas especies que tienen conductas del tipo de preferencias homosexuales (como en algunos primates, incluyendo los macacos japoneses). ${ }^{21}$

Este modelo, que sugiere que la evolución ha favorecido un mayor deseo sexual en los hombres, se apoya en las tasas de masturbación observados: no hay culturas humanas conocidas en las que las mujeres se masturben con más frecuencia que los varones. Lo mismo sucede con los primates no hu-

\footnotetext{
* Leer el libro El gen egoísta, de Richard Dawkings, para ampliar este concepto.
}

manos. Los monos Rhesus, criados en aislamiento de sus padres (para evitar un comportamiento aprendido), mostraron mayores tasas de masturbación en los machos, lo que sugiere una diferencia innata en la intensidad del deseo sexual. ${ }^{21}$

\section{TRANSEXUALIDAD}

\section{La experiencia subjetiva}

La transexualidad es el desarrollo de una identidad de género que está en desacuerdo con la morfología de los genitales y características sexuales secundarias. Se ha definido la identidad de género de la siguiente manera: "Es la propia categorización de la individualidad de uno como hombre, como una mujer o ambivalente". ${ }^{2}$ En la psiquiatría norteamericana se le sigue considerando una alteración mental bajo el rubro de "Disforia de género".

¿Cuál es el momento en el que un cuerpo aparece como parte del propio campo perceptual? Esta pregunta y varias más, sobre este mismo tema, son importantes y se encuentran relacionadas con lo que llamamos la estructura de la conciencia perceptiva. Este tipo de preguntas deben ser resueltas con tres enfoques: la fenomenología, la psicología y las neurociencias cognitivas.

\section{La conciencia de sí mismo como un comportamiento aprendido}

Los seres humanos tenemos en el cerebro una zona especializada que procesa la información sensorial adquirida por los órganos sensoriales. Los cinco sentidos conocidos para los seres humanos son la vista, el oído, el tacto, el gusto y el olfato (también conocido como los sentidos exteroceptivos, que también incluyen el equilibrio). Además de estos sentidos básicos, que proporcionan al cerebro información externa sobre el mundo, las conexiones neuronales en todo el cuerpo proporcionan al cerebro información sobre las diferentes partes que componen el organismo, ${ }^{3}$ así como las relaciones espaciales entre las diversas partes del cuerpo.

Un punto aparentemente obvio, que no se toma en cuenta, es que el cerebro se encuentra dentro de la cabeza del organismo, y que varios órganos sensoriales del organismo, así como conexiones sensoriales internas, están situados en partes distales y específicas del cuerpo, y que están comunicando al cerebro de una manera específica. Por lo tanto, el flujo de información desde el entorno siempre contiene un subconjunto constante de información relacionada con el organismo. Todo esto se lleva a cabo desde etapas tempranas de la gestación. El sentido del tacto, por ejemplo, detecta ya lo que es propio del cuerpo, y lo separa de lo extraño. La autopercepción se sigue desarrollando en los primeros años de vida. Un punto clave es cuando el niño puede identificar su imagen en el espejo y reconocerse como él mismo. ${ }^{3}$ 
Las personas transexuales también realizan este proceso. Lo interesante es que a pesar de que se identifican a sí mismos, hay una incoherencia con su percepción de género. Ésta se manifiesta de manera natural cuando se les pide que hagan tareas que culturalmente son asignadas a su género de nacimiento y hay un tipo de oposición a realizarlas. Esto los lleva a ser reprimidos, castigados, y hay una respuesta de asombro por parte de los menores, porque no entienden la incomprensión que despiertan en los demás por sus conductas.

\section{BASES BIOLÓGICAS DE LA TRANSEXUALIDAD}

Existen algunas hipótesis neurobiológicas acerca de esta condición humana; sin embargo, no se sabe en qué momento ocurren a lo largo de la gestación y cuáles son los factores que las condicionan. Las modificaciones en las estructuras cerebrales de la diversidad sexo-genérica están bien apoyadas por la biología y la genética. Hay variaciones en las estructuras cerebrales que expresan las conductas reproductivas, en especial en el hipotálamo.

Veale et al. ${ }^{27}$ revisaron los estudios previos de esta condición. Reportaron que hay evidencia de un componente genético de la diversidad sexo-genérica, con base en estudios con gemelos y otros dentro de la concordancia de la familia y de los estudios que han analizado específicamente cierto grupo de genes. También informaron de evidencia en los niveles de andrógenos prenatales, que se correlacionan con la diversidad de género, a partir de estudios de relaciones de longitud de los dedos (2D: 4D) de los transexuales, y de individuos que están más propensos a tener una identidad de género-variante o síndromes intersexuales y/o condiciones de ovario poliquístico. Además, existe evidencia de que los transexuales tienen algunas partes de su estructura cerebral que es más similar al sexo atípico, una mayor probabilidad de ser no-diestros, y mayor tendencia a reportar abuso infantil, y mientras que los transexuales, de hombre a mujer, a tener un mayor número de hermanos varones mayores. ${ }^{27}$

La relación entre la longitud de la segunda y la cuarta falanges de los dedos segundo y cuarto (2D: 4D) y la incapacidad de girar objetos de tres dimensiones mentalmente, se cree que son indicadores de exposición elevada a niveles de hormonas sexuales en etapas prenatales. ${ }^{27}$ Las causas de la lateralidad no-diestra (zurdos o ambidiestros) no se entienden completamente. Existe alguna evidencia acerca de que la zurdera se asocia con un aumento en los niveles de andrógenos prenatales y el desarrollo de una falta de dominancia hemisférica.

El elevado número de hermanos mayores que se encuentran en los transexuales de hombre a mujer (HaM), también se ha reportado en los hombres homosexuales. Se ha propuesto que cada feto del sexo masculino crea inmunización progresiva por los antígenos de los receptores a tes- tosterona, de tal manera que los próximos fetos masculinos tienen menos diferenciación de su cerebro hacia la virilización. ${ }^{28,29}$ Éste es un mecanismo de control poblacional, en el que, si bien no se puede controlar si el producto es hembra o macho, sí se actúa epigenéticamente, modificando sus capacidades reproductivas.

\section{EFECTOS DE LA ORGANIZACIÓN DE HORMONAS SEXUALES DURANTE ETAPAS TEMPRANAS DE DESARROLLO}

Las gónadas fetales se desarrollan bajo la influencia de una cascada de genes, que en los niños varones comienza con el gen determinante del sexo en el cromosoma Y (SRY). ${ }^{30} \mathrm{La}$ producción de testosterona y la conversión periférica de la testosterona en dihidrotestosterona, entre las semanas seis y 12 del embarazo, son esenciales para la formación del pene de un niño, de la próstata y del escroto. Sin embargo, el desarrollo de los órganos sexuales femeninos en el útero se debe principalmente a la ausencia de estos andrógenos. Una vez que la diferencia de estos órganos sexuales se cancela, la diferenciación sexual del cerebro ocurre, por efectos de la organización permanente, con las hormonas sexuales en el cerebro en desarrollo. ${ }^{31}$ Durante la pubertad, los circuitos cerebrales que se han organizado desde el periodo de estancia en la matriz serán activados por las hormonas sexuales, que hacen su irrupción en esta etapa de la aparición de caracteres sexuales secundarios.

El principal mecanismo responsable de la identidad de género y orientación sexual implica un efecto directo de la testosterona en el cerebro humano en desarrollo, como se muestra en los diferentes trastornos del tipo intersexual. El síndrome de insensibilidad completa a los andrógenos es causado por diferentes mutaciones en el gen para el receptor de andrógenos (AR). Los afectados son varones XY que se desarrollan como mujeres y tienen una apariencia fenotípica de mujer y fantasías "heterosexuales", sin los problemas de incoherencia de género. Cuando un feto varón tiene una deficiencia de 5 alfa-reductasa-2 o $17 \beta$ hidroxi-esteroides deshidrogenasa-3, ocurre que la testosterona periférica se transforma en dihidrotestosterona. Al nacer, se presenta como una "niña" con un clítoris grande. Estos niños XY son generalmente criados como niñas. Sin embargo, cuando aumenta la producción de testosterona durante la pubertad, el 'clítoris' crece al tamaño de un pene, los testículos descienden, y los niños comienzan a masculinizarse y se hacen más musculosos.

\section{CONCLUSIÓN}

Nuestra especie está organizada de manera natural como el resto de las especies animales. Sin embargo, la cultura crea un orden no natural. Es importante conocer el estado 
de estos temas, que a fuerza de repetirse crean la ilusión de "lo normal". En su libro La dominación masculina, Pierre Bourdieu $^{32}$ matiza lo expresado anteriormente diciendo: "hay una visión del mundo con la que el hombre (el varón), satisface su sed de dominio, una visión que las propias mujeres (y personas de la diversidad sexo-genérica), sus propias víctimas, han asumido, aceptando inconscientemente su inferioridad". Lo anterior está aceptado como inherente a nuestras formas culturales. Bourdieu continúa: "La fuerza del orden masculino se descubre en el hecho de que prescinde de cualquier justificación, la visión androcéntrica se impone como neutra y no siente la necesidad de enunciarse en unos discursos capaces de legitimarla. El orden social funciona como una inmensa máquina simbólica que tiende a ratificar la dominación masculina en la que se apoya: es la división sexual del trabajo, distribución muy estricta de las actividades asignadas a cada uno de los dos sexos (géneros), de su espacio, su momento, sus instrumentos". ${ }^{32}$

Lo dicho por este filósofo francés se puede extrapolar al grupo de la diversidad sexo-genérica. El aprender que éstas son condiciones naturales, que no se eligen, y que sin embargo han sido reprimidas y perseguidas por las instituciones religiosas, políticas y médicas, sirve para ubicarnos desde una perspectiva diferente. Dentro de la teoría general evolutiva hay explicaciones para integrar la diversidad, no sólo sexo-genérica, sino otras, como la sinestesia, las variaciones del carácter, la resistencia o permisividad para afrontar los cambios, la resiliencia ante la adversidad, la capacidad para detectar memes y el sentido del humor. Somos una especie compleja, pero en la que la diferencia es la norma. De no verlo así, seguiremos siendo los tuertos del mundo animal.

\section{Financiamiento}

Ninguno.

\section{Conflicto de intereses}

El autor declara no tener conflicto de intereses.

\section{REFERENCIAS}

1. Gallarda T, Amado I, Coussinoux S, Poirier MF et al. The transsexualism syndrome: clinical aspects and therapeutic prospects. L'Encephale 1997;23:321-326.

2. Herman-Jeglinska A, Grabowska A, Dulko S. Masculinity, femininity, and transsexualism. Archives Sexual Behavior. 2002;31:527-534.

3. Bodlund $\mathrm{O}$, Armelius K. Self-image and personality traits in gender identity disorders: an empirical study. J of sex Marital Therapy. 1994;20:303-317.

4. Lemma A. The body one has and the body one is: understanding the transsexual's need to be seen. The International J Psycho-analysis 2013;94:277-292.

5. Kraemer B, Delsignore A, Schnyder U, Hepp U. Body image and transsexualism. Psychopathology 2008;41:96-100.

6. Cozolino LJ. The neuroscience of human relationships : attachment and the developing social brain. Segunda edición. Nueva York, Norton \& Company; 2014.
7. Meyenburg B, Sigusch V. Kallmann's syndrome and transsexualism. Archives Sexual Behavior 2001;30:75-81.

8. Kim KS, Kim J. Disorders of sex development. Korean J Urology 2012;53:1-8.

9. Hughes IA. Disorders of sex development: a new definition and classification. Best Practice Research Clinical Endocrinology Metabolism 2008;22:119-134.

10. Wierckx K, Van Caenegem E, Pennings G, Elaut E et al. Reproductive wish in transsexual men. Human Reproduction. 2012;27:483-487.

11. Beach FA. Animal models for human sexuality. Ciba Foundation Symposium; 1978; pp.113-143.

12. Bailey NW. Evolutionary models of extended phenotypes. Trends Ecology Evolution 2012;27:561-569.

13. Bailey NW, Macias Garcia C, Ritchie MG. Beyond the point of no return? A comparison of genetic diversity in captive and wild populations of two nearly extinct species of Goodeid fish reveals that one is inbred in the wild. Heredity 2007;98:360-367.

14. Bailey NW, Zuk M. Same-sex sexual behavior and evolution. Trends Ecology Evolution 2009;24:439-446.

15. Bailey NW, Zuk M. Field crickets change mating preferences using remembered social information. Biology (letters) 2009;5:449-451.

16. Sommer V, Vasey PL. Homosexual behaviour in animals : an evolutionary perspective. Cambridge; Nueva York: Cambridge University Press; 2006.

17. Harris AL, Vitzthum VJ. Darwin's legacy: an evolutionary view of women's reproductive and sexual functioning. J Sex Research 2013;50:207-246.

18. Harts AM, Kokko H. Understanding promiscuity: when is seeking additional mates better than guarding an already found one? Evolution. International J Organic Evolution 2013;67:2838-2848.

19. Zuk M, Blley NW. Birds gone wild: same-sex parenting in albatross. Trends Ecol Evol 2008;2008:3.

20. Yaung LC, Zaun, BJ, Wanderwerf EA. Successful same-sex pairing in Laysan albatross. Biol Lett;4:3.

21. Gray PB. Evolution and human sexuality. American J Physical Anthropology 2013;152(Suppl)57:94-118.

22. Rice WR, Friberg U, Gavrilets S. Homosexuality via canalized sexual development: a testing protocol for a new epigenetic model. BioEssays: news and reviews in molecular, cellular and developmental biology. 2013;35:764-770.

23. Jensen GD, Kales J, Abernethy V. Letter: Dominance, sexual preference, and sexism. American J Psychiatry 1974;131:1413-1414.

24. Mainardi D, Mainardi M. Ethology and veterinary science. Folia Veterinaria Latina 1977;7:295-306.

25. Abernethy V. Dominance and sexual behavior: a hypothesis. American J Psychiatry. 1974;131:813-817.

26. Williams JB. Homosexuality in nonhuman primates: a bibliography, 1940-1992. Seattle, Wash.: Primate Information Center, Regional Primate Research Center, University of Washington; 1992.

27. Veale JF. Prevalence of transsexualism among New Zealand passport holders. Australian New Zealand J Psychiatry 2008;42:887-889.

28. Blanchard $R$. The concept of autogynephilia and the typology of male gender dysphoria. J Nervous Mental Disease. 1989;177:616-623.

29. Blanchard R, Clemmensen LH, Steiner BW. Social desirability response set and systematic distortion in the self-report of adult male gender patients. Archives Sexual Behavior 1985;14:505-516.

30. Lombardo F, Toselli L, Grassetti D, Paoli D. Hormone and genetic study in male to female transsexual patients. J Endocrinological Investigation 2013;36:550-557.

31. Cohen-Kettenis PT, van Goozen SH, Doorn CD, Gooren LJ. Cognitive ability and cerebral lateralisation in transsexuals. Psychoneuroendocrinology 1998;23:631-641.

32. Bourdieu P. La domination masculine. París: Editions du Seuil; 1998. 